\title{
Nano-JASMINE: a nano size astrometry satellite
}

\author{
Yukiyasu Kobayashi ${ }^{1}$, Taihei Yano ${ }^{1}$, Naoteru Gouda ${ }^{1}$, Yoshiyuki \\ Yamada $^{2}$, Naruhisa Takato ${ }^{1}$, Satoshi Miyazaki ${ }^{1}$, Masahiro Suganuma ${ }^{1}$, \\ Seiji Ueda ${ }^{4}$, Shin'ichi Nakasuka ${ }^{3}$, and the JASMINE working group \\ ${ }^{1}$ National Astronomical Observatory, 2-21-1 Osawa Mitaka Tokyo 181-8588, Japan \\ email:yuki@merope.mtk.nao.ac.jp,t.yano@nao.ac.jp,naoteru.gouda@nao.ac.jp, \\ takato@subaru.naoj.org, satoshi@naoj.org, suganuma@merope.mtk.nao.ac.jp \\ ${ }^{2}$ Department of Physics, Kyoto University, Kyoto 606-8502, Japan \\ email:yamada@amesh.org \\ ${ }^{3}$ The Graduate University for Advanced Studies, 2-21-1 Osawa Mitaka Tokyo Japan \\ email: seiji.ueda@nao.ac.jp \\ ${ }^{4}$ Faculty of Engineering, The University of Tokyo, 7-3-1 Hongo Bunkyo-ku Tokyo Japan \\ email:nakasuka@space.t.u-tokyo.ac.jp
}

\begin{abstract}
We present the outline and current status of the nano-JASMINE project. NanoJASMINE is a nano size astrometry satellite (the total payload mass is between $1 \mathrm{~kg}$ and $10 \mathrm{~kg}$ ), which is expected to be launched in 2006. The main purpose of the project is to prove and demonstrate the key technologies required for JASMINE (Japanese Astrometry Satellite Mission for Infrared Exploration) in a real space environment Gouda et. al. (2002). Nano-JASMINE will measure annual parallaxes of bright stars $(7 \mathrm{mag})$ with an accuracy greater than 1 milliarcsecond after two years of operation. This is comparable to the accuracy of the Hipparcos catalog. Currently, the subject of major research and development of the project is to build optical and telescope structures using new material, and to establish a data acquisition and control system.
\end{abstract}

\section{Mission Target}

Nano-JASMINE is a very small satellite that will be used to demonstrate and verify new technologies developed for the JASMINE project. JASMINE will carry out astrometry observations for stars located in a narrow strip within $4^{\circ}$ of the Galactic plane at the z-band (central wavelength of $0.95 \mu \mathrm{m}$ ). JASMINE will measure the position, annual parallax and proper motion of these stars with accuracies greater than ten microarcseconds. By measuring the motion of millions of stars, both deep in the Galactic plane and in the central bulge region, JASMINE will reveal the dynamic evolution of the Galaxy. Its launch is planned within the next ten years, and vigorous technical research and development is currently underway. These includes TDI control of an array detector, ultra-light mirror, electronic components for space radiative environment, techniques on thermal deformation and ultra precision position measurements. Nano-JASMINE will demonstrates and verify these key technologies in real space environments. At the same time, it will make clear the future problems that JASMINE might face. Thus, this would be a good exercise for the JASMINE team. Using nano-JASMINE, we can test new technologies in a real space environment during the development stage itself. The advantages of using a very small satellite, such as a nano-sized satellite is that its cost is not very high and the results can be realized in a short span of time. Furthermore, we can use the unoccupied space of a launcher; this makes the chance of a launch high and its cost low. 


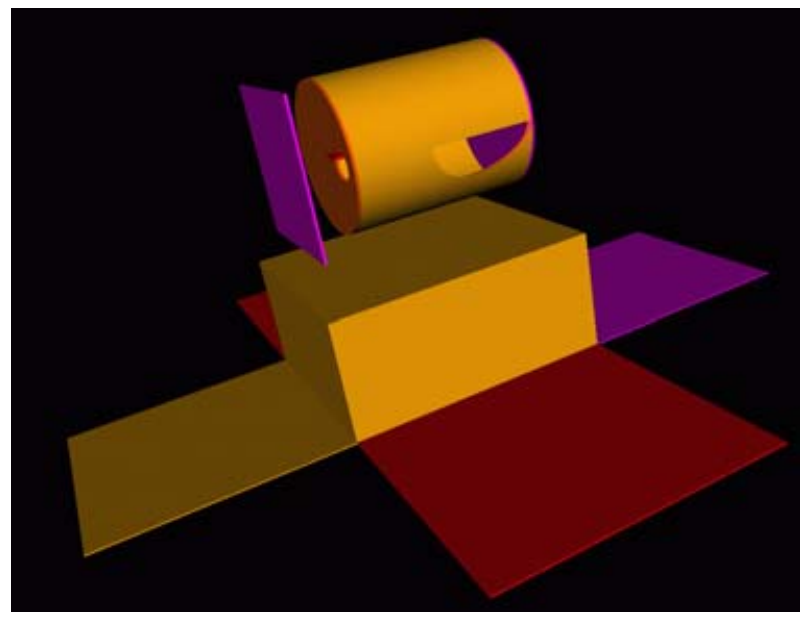

Figure 1. Overall view of nano-JASMINE.

Table 1. General Features of nano-JASMINE

\begin{tabular}{ll}
\hline Orbit & $\begin{array}{l}\text { Sun-synchronous } \\
\text { Spin rate }\end{array}$ \\
Height & $300 \mathrm{~km}-800 \mathrm{~km}$ \\
& \\
Diameter & $5 \mathrm{~cm}$ \\
Focus Length & $167 \mathrm{~cm}$ \\
Field of View & $30^{\prime} \times 30^{\prime}$ \\
Basic Angle & 99.5 \\
& \\
Detector & Full depletion CCD \\
Pixel Format & $1024 \times 1024$ \\
Pixel Size & $15 \mu \mathrm{m}$ \\
\hline
\end{tabular}

\section{Mission Plan}

The first nano-JASMINE satellite is expected to be launched in 2006 into a sunsynchronous orbit at an altitude of between $300 \mathrm{~km}$ and $800 \mathrm{~km}$. The total payload mass of nano-JASMINE is a few kilograms. A satellite that weighs between $1 \mathrm{~kg}$ and $10 \mathrm{~kg}$ is usually called a nano-satellite. Thus, we have named this satellite nano-JASMINE. We will employ a nano-satellite, because of its high chance of launch and because it is capable of supporting an appropriate level of astrometry observations. In order to obtain very precise position information, we simultaneously observe two fields in a great circle with a large separation angle. We expect an accuracy of one milliarcsecond for stars in a certain great circle after one year of operation. The selection of the great circle depends on the launch conditions. The maximum data rate will be of the order of $5000 \mathrm{kbps}$. We use the z-band filter (central wavelength of $0.95 \mu \mathrm{m}$ ), the center of which is the longest part of the silicon CCD's sensitive wavelength region. The satellite will spin at a rate of one revolution every forty minutes in order to conduct a survey along the concerned great circle.

\section{Telescope and Optics}

The nano-JASMINE telescope has a diameter of $5 \mathrm{~cm}$ with a beam combiner placed in front of the primary mirror. We adopt the Korsh optical system. This system consists 


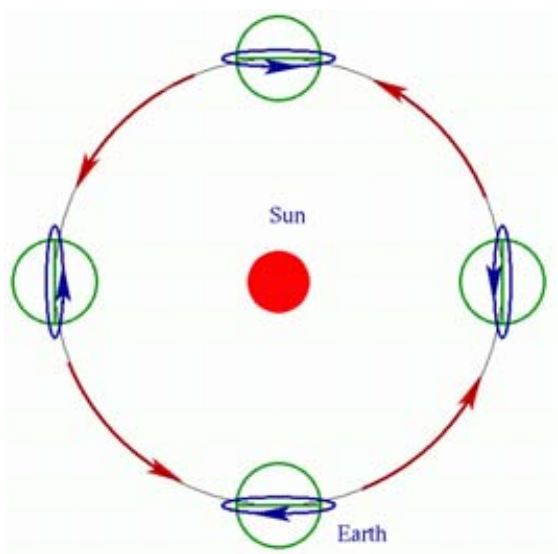

Figure 2. Sun-synchronous Orbit.

of three mirrors that provide a flat focal plane and a wide field-of-view. With a focal length of $167 \mathrm{~cm}$ the telescope provides a diffraction limited image which fills two pixels at the focal plane. Basically, the optical system used is a scaled down version of that of JASMINE; however, some modifications are required to solve the problems that arise when optical components become very small. Fig. 1 shows the complete view of the optical layout. Two flat mirrors, angled at $49^{\circ}$, and placed in front of the primary mirror allow the system to simultaneously observe two fields separated by an angle of $95^{\circ}$. Deformation of the optical components due to thermal variations in the environment would make the achievement of high precision astrometry observations almost impossible. The structure and optics are constructed using the same material to reduce thermal deformation. Currently, we concentrate on the research and development of two kinds of materials. The first material is aluminum. Employing modern super-precise machines, it is possible to construct a telescope using aluminum optics and structure. This type of telescope is suitable for nano-JASMINE. The other material under consideration is reaction sintered silicon carbide developed by Toshiba Tsuno et. al. (2004). Reaction sintered silicon carbide is manufactured by reaction sintering molten silicon to spray dried SiC Carbon powder. It has high thermal stability and favorable properties for space telescope material. The reason behind developing the nano-JASMINE telescope using reaction sintered $\mathrm{SiC}$ is that this is one of the best candidate materials used to construct the JASMINE telescope.
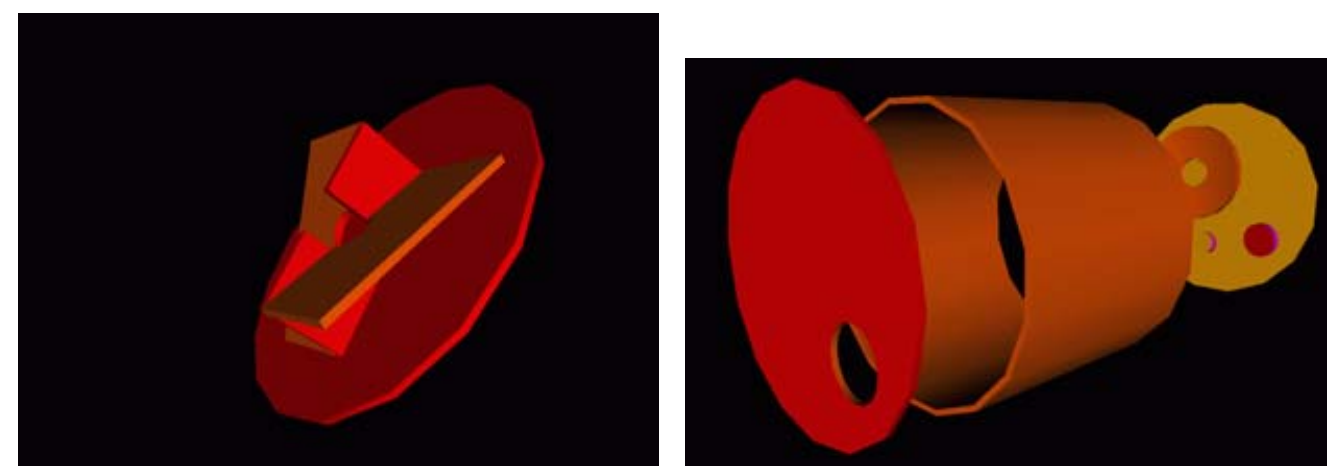

Figure 3. Telescope structure design. 


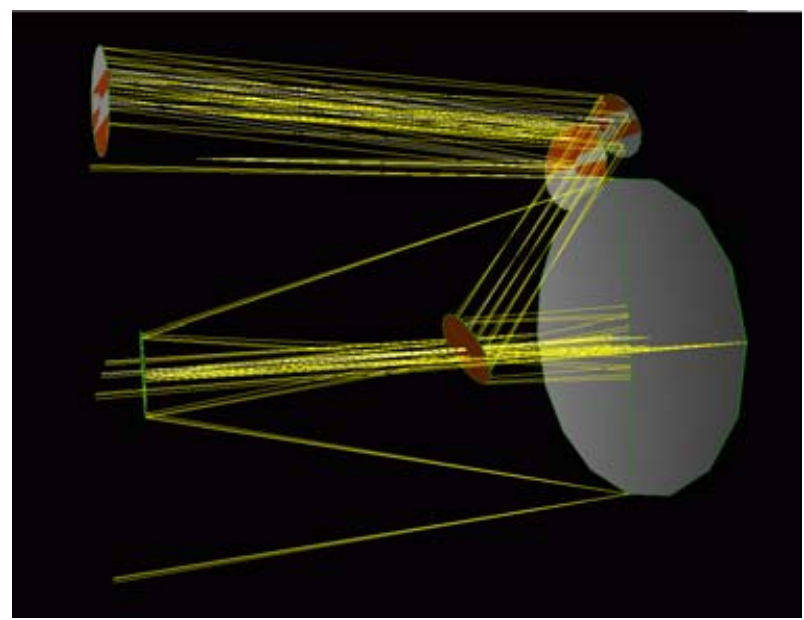

Figure 4. Optical layout.

\section{CCD and Control System}

For the purpose of survey observations, the CCD and control system of nano-JASMINE use a $1024 \times 1024$ format, full depletion CCD, operated in TDI mode. The pixel size is $15 \mu \mathrm{m}$, which is equivalent to $11^{\prime \prime} 8$ projected on the sky. We are developing our own readout and controlling system. The full depletion CCD has a high response at longer wavelengths. The National Astronomical Observatory and Hamamatsu Photonics are developing the same type of CCD for the new Subaru instrument. We will manufacture the same type of CCD with a smaller format for nano-JASMINE, with a total field of view of $30^{\prime} \times 30^{\prime}$. In order to make the readout noise low at a high read out frequency, the CCD has two output channels. A 3-s integration time is employed under the TDI operation. Fig. 2 shows the readout circuit board, which has two 16 bit AD channels with a 3 mus sampling rate. The TDI scan rate must be adjusted according to the satellite spin rate; the CCD controller is equipped to accept a fine adjust control command to change the scan rate. The digitized data is picked up and saved as single stellar information. This part of the operation is realized as hardware using FPGA. This is because it requires high speed processing; however, it has a relatively simple processing procedure. After receiving data from FPGA, the system controller carries out the calibration and compresses the data to reduce the data size. In order to synchronize the TDI scan rate with the satellite
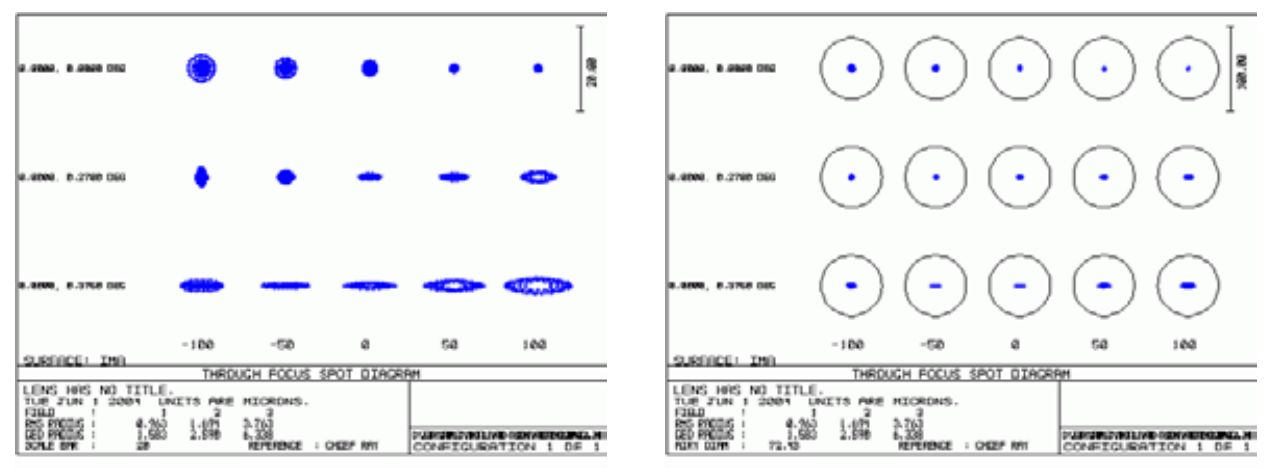

Figure 5. Optical system spot diagram. 


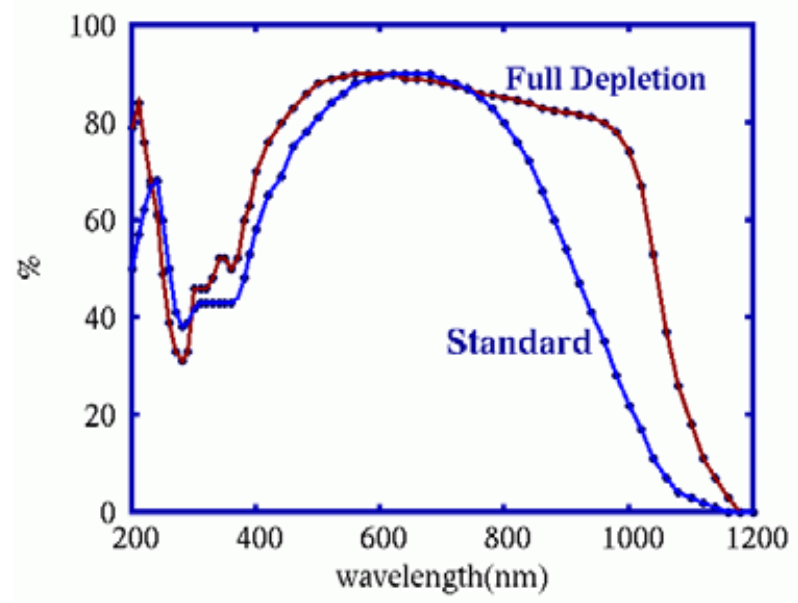

Figure 6. Spectral response of the full depletion CCD.

spin rate, stellar image data is analyzed by the system controller and feedback is sent to the posture control system and a scan shift command is sent to the CCD controller.

\section{Bus System}

The new bus system, based on the PRISM (Pico-satellite for Remote sensing and Innovative Space Missions ) system, will be developed for nano-JASMINE. PRISM is a system that has been under development at the University of Tokyo as a second generation system based on the Cube-Sat XI-V. PRISM will realize a higher performance satellite mission based on a very small satellite system. The system offers a 3-axis controller, equipped with a magnetic torquer and a reaction wheel. It also includes a command and data processing system, power supply and a telecommunication system. Although it is a simple system, it is capable of performing several tasks.

\section{Conclusion}

As a facility to demonstrate and verify new technologies in real space environments, a nano-sized satellite is very useful. It provides the opportunity of conducting experiments in a short span of time at low costs. In fact, realizing a real space environment is difficult given the existing ground-based facilities. If the equipment commonly used for such small satellites is fully prepared, it would serve as a powerful platform for many other applications.

\section{References}

Gouda N., Tsujimoto T., Kobayashi Y., Nakajima T., Matsuhara H., Yano T., Yasuda N., KanYa Y., Yamada Y., Ueno M. 2002 The Proceedings of the IAU 8th Asian-Pacific Regional Meeting Volume II, Edited by S. Ikeuchi, J. Hearnshaw, and T. Hanawa , 5-6

Tsuno Bearman P.W., Graham J.M.R. 2004 Proceedings of 5th Internatinal Conference on Space Optics 2004, Toulouse. 